\title{
Capillary condensation transitions in a slab geometry
}

\author{
Silvina M. Gatica, ${ }^{1,2}$ M. Mercedes Calbi, ${ }^{1,2}$ and Milton W. Cole ${ }^{1, *}$ \\ ${ }^{1}$ Department of Physics, Penn State University, University Park, Pennsylvania 16802 \\ ${ }^{2}$ Departamento de Fisica, Facultad de Ciencias Exactas y Naturales, Universidad de Buenos Aires, 1428 Buenos Aires, Argentina
}

(Received 2 October 1998)

\begin{abstract}
A simple model, previously used to explore wetting transitions, is evaluated for the case of a slab geometry in which adsorption occurs between two semi-infinite solids, with parallel faces separated by a distance $L$. The model yields a universal description of possible wetting and capillary condensation (CC) transitions. The system's thermodynamic behavior is predicted from the values of two dimensionless parameters: $D^{*}$ (the reduced gas-surface interaction strength, a function of temperature) and $L^{*}$ (the reduced separation). If $D^{*}$ $<2.9$, negligible adsorption occurs at all pressures below saturated vapor pressure (SVP). For somewhat larger values of $D^{*}, \mathrm{CC}$ occurs for sufficiently small $L^{*}$ close to SVP. For very large values of $D^{*}$, an additional prewetting transition (formation of a film) is predicted for large $L^{*}$; this is accompanied by a CC transition close to SVP. The model is generally consistent with limited results of density-functional calculations for the He liquids at zero temperature. [S1063-651X(99)10804-3]
\end{abstract}

PACS number(s): 68.10. $-\mathrm{m}, 68.15 .+\mathrm{e}, 67.70 .+\mathrm{n}$

\section{INTRODUCTION}

Capillary condensation, the condensation in small pores of liquid at ambient pressure below saturation, has long been known to occur [1-9]. In some cases, this behavior can be understood adequately in terms of the Kelvin equation (KE), which expresses the adsorption below saturated vapor pressure (SVP) in terms of the surface tension $(\sigma)$ and radius $R$ of the pore, assuming typically a cylindrical geometry for simplicity. However, the KE description has long been known to fail quantitatively (and even qualitatively in some circumstances) for small pores. The reason is that its simplicity is a result of sacrificing the role of gas-surface interactions, which can be crucially important in wetting problems.

For wide pores and a strongly attracting host medium, the error in using the KE is small; then the KE can be corrected by subtracting from $R$ the thickness of the film prior to the capillary condensation CC transition. This approach does not suffice if the adsorption interaction is weak and/or $R$ is small. It is, therefore, useful to seek an alternative formulation, which predicts more accurately and generally the wide range of behavior which can occur.

In this paper we present such a simple model of CC for a "slab" geometry in which the adsorption space is bounded by two semi-infinite solids, whose surfaces are parallel planes separated by a distance $L$. Like the KE, our model is derived from a simple set of assumptions and, therefore, sacrifices some degree of quantitative accuracy. The approach used here is analogous to one which we have used in studies of wetting transitions. The predictions have been tested in that context by comparing the results with both reliable (i.e., "exact" Monte Carlo classical and density-functional quantum) calculations, and even experiments [10-14]. The method has proven to be accurate in general. That success has encouraged us to extend the method in the present paper.

\footnotetext{
*Author to whom correspondence should be addressed. Electronic address: mwc@psu.edu
}

The outline of this paper is the following. In the next section we introduce the model and show that it leads to a single equation from which the behavior can be deduced for each of the possible phases that may be present. That equation expresses a reduced grand free energy $\Omega^{*}$ in terms of three reduced dimensionless variables: the strength $D^{*}$ of the adsorption potential, the separation $L^{*}$ between the confining walls, and $\Delta$, the chemical potential difference from SVP. (Another relevant variable, temperature $T$, plays an implicit role in the determination of the reduced variables.) From the minimum value of $\Omega^{*}$ one predicts which of three possible phases is present. These include an "empty" phase, meaning negligible adsorption, a "film" phase, meaning multilayer film adsorption, and a capillary condensed phase, which fills the space with liquid. This approach leads to a generalized "phase diagram," which expresses the predicted behavior in terms of two of the possible three variables (at fixed value of the third); see, e.g., Fig. 1. While a three-dimensional representation of the behavior is possible, we have found that to be less useful than a set of two-dimensional figures.

This paper extends and generalizes the method applied by Cheng, Swift, and Cole some years ago [8]. In Sec. III, we compare the results of our present simple model with their results of density-functional $[15,16]$ calculations for ${ }^{4} \mathrm{He}$ and with new results for ${ }^{3} \mathrm{He}[12,17]$. The agreement overall is quite good. In future publications, we will present more extensive and general results, currently being obtained with reliable methods for both classical and quantum systems. We note that our simple model is not capable of describing other important transition phenomena, such as layering transitions, which have been studied previously with more accurate models [18].

\section{MODEL CALCULATIONS}

\section{A. General discussion}

Throughout our analysis we have employed a 3-9 form of the adsorption potential arising from each of the solids bounding the adsorption domain: 


$$
V(z)=\left\{4 C^{3} /\left[27 D^{2}\right]\right\} z^{-9}-C z^{-3} .
$$

Here the atom lies at distance $z$ from the image plane position, a quantity which has been computed and measured experimentally [19]. This functional form (used in much previous work on wetting) captures the key aspects of the potential and includes parameters, which are relatively well known in many cases: $D$ is the well depth and $C$ is the strength of the van der Waals asymptotic interaction [19]. For this potential, the equilibrium distance is

$$
z_{m}=[2 C /(3 D)]^{1 / 3} .
$$

The net potential in the slab geometry is taken to be a sum of the respective contributions from the two surfaces [20]. The adsorption behavior is to be predicted by evaluating the equilibrium number of atoms adsorbed per unit area $N_{\text {eq }}$ as a function of chemical potential $\mu$. The value of $N_{\text {eq }}$ and the other thermodynamic properties may be determined by minimizing (as a function of variable $N$ ) the grand potential per unit area,

$$
\Omega(N)=F(N)-\mu N,
$$

where $F$ is the Helmholtz free energy per unit area [21]. The value of $T$ is implicitly present in both $F$ and $\Omega$. One may, in principle, carry out this minimization procedure exactly by Monte Carlo methods (except for metastable or unstable ranges of parameters), but here we exploit instead a very simple model. The model assumes that the solid-liquid interfacial tension consists of the sum of the bulk liquid-vapor interfacial tension $\sigma$ and the integral of the gas-surface interaction over the region occupied by liquid (which has a number density $n$ ). This really ought to be supplemented by twice the surface tension of the bare substrate, but we omit that additive quantity throughout this paper.

For the sake of generality of application, we discuss our predictions in terms of dimensionless quantities. These include a reduced grand potential, gap distance, chemical potential relative to its value $\left(\mu_{0}\right)$ at SVP, and well depth, defined as

$$
\begin{gathered}
\Omega^{*}=\Omega / \sigma, \\
L^{*}=L / z_{m}, \\
\Delta=\left(\mu_{0}-\mu\right) n z_{m} / \sigma, \\
D^{*}=2 n D z_{m} / \sigma .
\end{gathered}
$$

Table I presents values of $z_{m}$ and $D^{*}$ for selected systems of interest (using the "best'" values of the potential energy parameters); one should bear in mind that the values of $D^{*}$ increase with $T$, thus increasing the tendency to condense. Note the extended range of values of $D^{*}$, indicating a wide variety of possible behaviors. Note in particular the strong tendency of the He liquids to condense; their large values of $D^{*}$ arise from the small ratio of surface tension to density. Another dimensionless quantity is an integral:

$$
g(y)=\int d x\left[-V\left(z_{m} x\right) / D\right]
$$

TABLE I. The values of $z_{m}$ (in $\AA$ ) and dimensionless well depth $D^{*}$, in parentheses, for inert gases and $\mathrm{H}_{2}$ on various surfaces. The original data are found in Refs. [10], [14], and [19]. The temperatures are $T=0$ for ${ }^{3} \mathrm{He}$ and ${ }^{4} \mathrm{He}$, and $T_{\mathrm{tp}}$ for the other gases.

\begin{tabular}{lcccc}
\hline \hline Gases & $\mathrm{Cs}$ & $\mathrm{Li}$ & $\mathrm{Au}$ & Graphite \\
\hline${ }^{3} \mathrm{He}$ & $4.55(9.42)$ & $3.95(20.9)$ & $2.66(88.8)$ & $1.93(110)$ \\
${ }^{4} \mathrm{He}$ & $4.55(5.50)$ & $3.95(12.2)$ & $2.66(51.8)$ & $1.93(64.4)$ \\
$\mathrm{H}_{2}$ & $3.66(3.33)$ & $3.21(7.71)$ & $2.34(26.1)$ & $1.86(26.8)$ \\
$\mathrm{Ne}$ & $3.55(1.55)$ & $3.40(3.17)$ & $2.48(12.8)$ & $1.92(13.4)$ \\
$\mathrm{Ar}$ & $2.78(1.60)$ & $3.02(3.22)$ & $2.40(10.2)$ & $2.03(9.77)$ \\
$\mathrm{Kr}$ & $2.56(1.70)$ & $2.91(3.40)$ & $2.39(9.99)$ & $2.10(9.15)$ \\
$\mathrm{Xe}$ & $2.59(1.68)$ & $2.88(3.46)$ & $2.41(9.28)$ & $2.16(8.06)$ \\
\hline \hline
\end{tabular}

with the lower limit set equal to unity and the upper set equal to $y$. This function is positive for the relevant regime $(y$ $>1$ ) and has an asymptotic behavior,

$$
g(y) \sim 11 / 16-3 /\left(4 y^{2}\right) \quad(y \gg 1) .
$$

With this general approach and notation, we may evaluate the grand potential for any hypothetical film configuration. Figures 1-6 present the thermodynamically stable phases derived from this approach. As exemplified in Fig. 1, there is a regime of parameter space that exhibits no adsorption $(\Omega *$ $>0$ ), a regime where a film is stable, and a capillary condensed CC region.

\section{B. Capillary condensed phase}

If the liquid is capillary condensed, the grand free energy per unit area is taken to be

$$
\Omega_{\mathrm{CC}}=2 \sigma+2 n \int d z V(z)-\left(\mu-\mu_{0}\right) n\left(L-2 z_{m}\right) .
$$

Here, the integration domain extends from $z_{m}$ to $L-z_{m}$, since that is the region occupied by (approximately uniform density) fluid. The factor of 2 multiplying the integral ap-

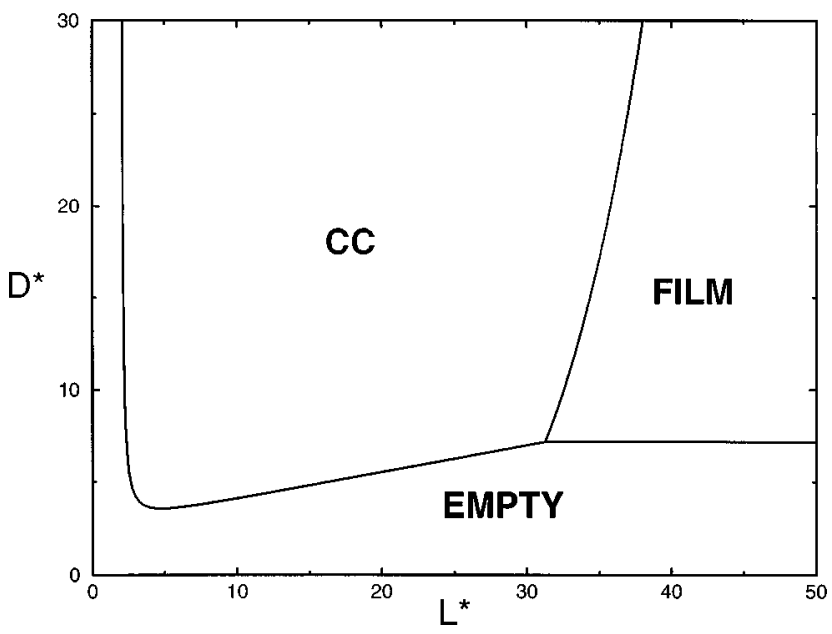

FIG. 1. Universal "phase diagram" showing regimes of empty, capillary condensed, and adsorbed film as a function of reduced well depth and gap spacing, defined in the text, for the case of reduced chemical potential $\Delta=0.1$. 


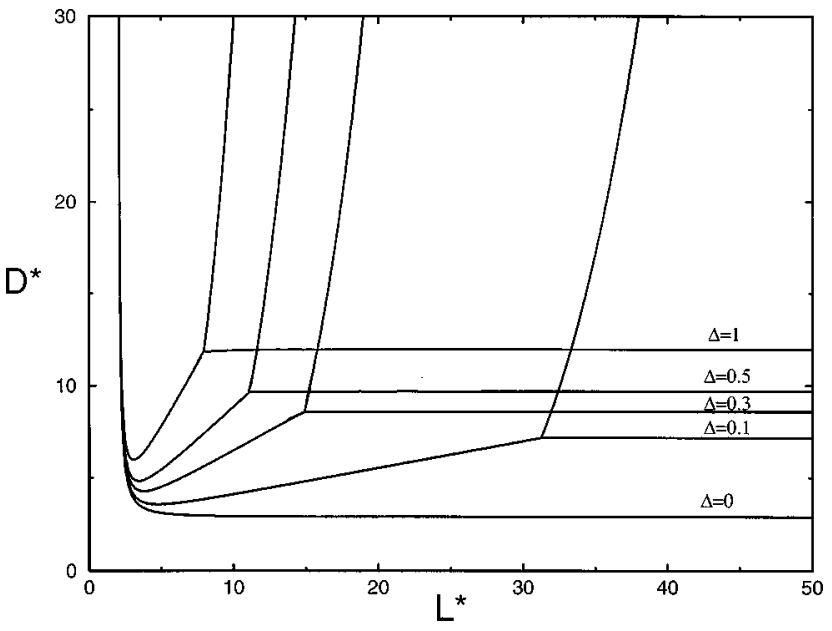

FIG. 2. Curves analogous to those in Fig. 1, exhibiting phases present at varying degrees of undersaturation, expressed in terms of the reduced chemical potential difference $\Delta$. For the case $\Delta=0$, the line satisfies Eq. (7); all values above the curve correspond to the case of capillary condensation, while those below are "empty." For the other cases, there is a "triple point" below which the space is empty, to the upper right of which there is a film, and to the upper left of which there is capillary condensation (as in Fig. 1).

pears because we assume that the total potential energy is the sum of contributions from each surface, i.e., $V(z)+V(z$ $-L)$. While this approach is analogous to that used previously to describe wetting transitions, the detailed calculation involves some differences: in the wetting case, the factor of 2 is absent and the integral's upper limit is infinity when computing the wetting transition, because an infinitely thick film appears at that transition [10]. From Eq. (5), we may "derive'" the modified Kelvin equation for $\mathrm{CC}$,

$$
0=2 \sigma_{1 s}+\left(P-P_{0}\right)\left(L-2 z_{m}\right)
$$

by setting $\Omega_{\mathrm{CC}}=0$, writing the first two terms of Eq. (5) as twice the substrate-liquid interfacial tension $\sigma_{l s}$ and using

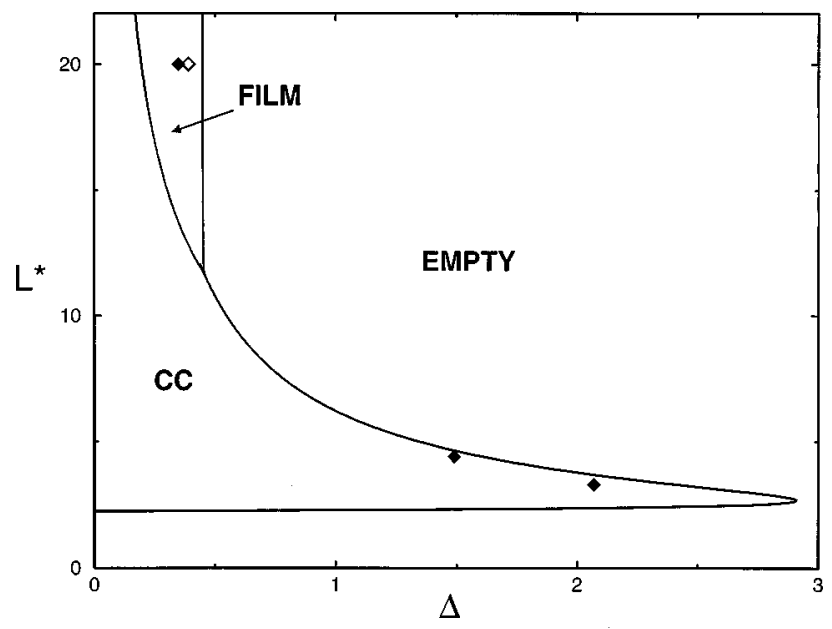

FIG. 3. Reduced phase diagram for $D^{*}=9.42$ (e.g., ${ }^{3} \mathrm{He}$ on Cs at $T=0)$. The curves are calculated with the present model. The diamonds indicate the prewetting transition results of Rutledge and Taborek [11] (open), which are consistent with density-functional results of Calbi et al. (closed).

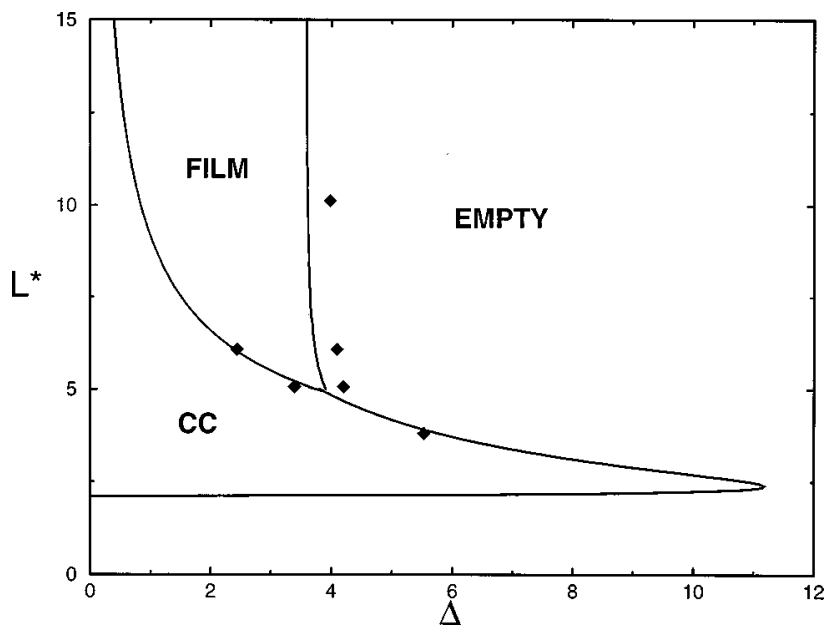

FIG. 4. Same as Fig. 3, except that $D^{*}=20.9$ (e.g., ${ }^{3} \mathrm{He}$ on $\mathrm{Li}$ at $T=0)$.

the Gibbs-Duhem [21] relation for the liquid, $\left[\left(\mu-\mu_{0}\right) n\right.$ $\left.=\left(P-P_{0}\right)\right]$, neglecting any compression of the film. Equation (6) expresses the pressure reduction for condensation in terms of the effective gap, $\left(L-2 z_{m}\right)$. This paper's goal is to evaluate both the explicit dependence on the substrate potential and the role of film formation on the adsorption behavior, thus generalizing the KE.

From explicit integration of Eq. (5), using Eq. (4) we obtain an expression for the reduced grand potential,

$$
\Omega_{\mathrm{CC}} *=2-D^{*} g\left(L^{*}-1\right)+\Delta\left(L^{*}-2\right),
$$

The case of SVP yields a particularly simple criterion for CC to occur. The resulting universal transition line,

$$
D^{*}=2 /\left(g L^{*}-1\right),
$$

appears in Fig. $2(\Delta=0)$. That curve separates the behavior into two regimes: capillary condensation $\left(\Omega_{\mathrm{CC} *}<0\right)$ or empty $\left(\Omega_{\mathrm{CC} *}>0\right)$. This provides an explicit dependence on the values of the parameters $L^{*}$ and $D^{*}$. One observes a possibly surprising behavior: the threshold value of $D^{*}$ (above which CC occurs) decreases as $L^{*}$ increases (since $g$

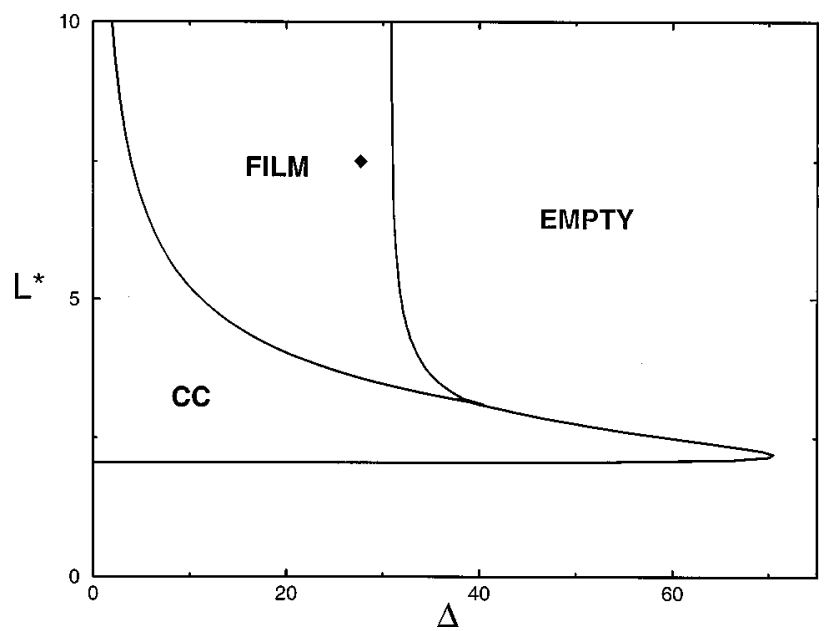

FIG. 5. Same as Fig. 3, except that $D^{*}=88.8$ (e.g., ${ }^{3} \mathrm{He}$ on $\mathrm{Au}$ at $T=0)$. 


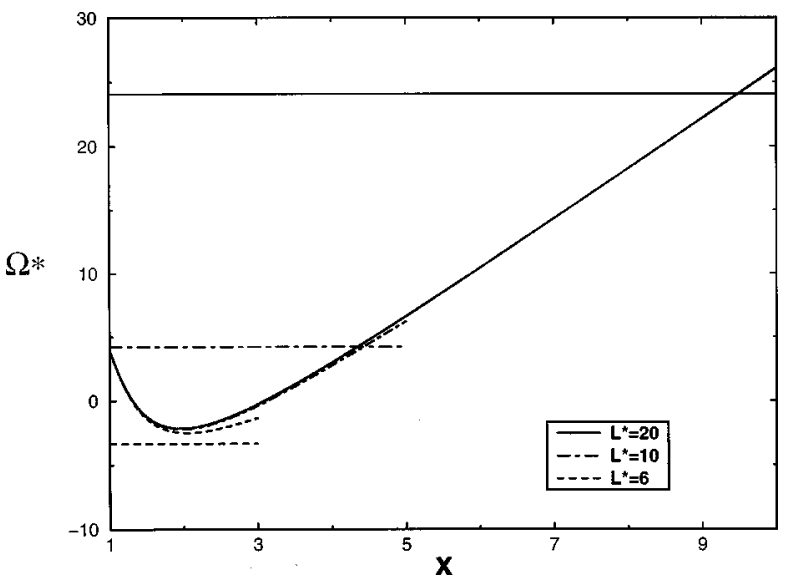

FIG. 6. Dimensionless grand potentials $\Omega_{F}^{*}$ at $\Delta=2$, for films of various (hypothetical) reduced thickness $(x)$ of ${ }^{3} \mathrm{He}$ on Li (curved lines), to be compared with values of $\Omega_{\mathrm{CC} *}$ (horizontal lines). The curves correspond to different values of $L^{*}$ as shown in the inset. Only for $L^{*}=6$ is the CC phase stable, since that curve is lowest. In the case of $L^{*}=10$ and 20, the film is stable and the equilibrium thickness is found from the minimum of $\Omega_{F}^{*}$ as a function of $x$.

is a monotonic function). The reason why this may be a surprise is that $\mathrm{CC}$ is typically found in small pores rather than large pores, while the case $\Delta=0$ shows the opposite trend. Indeed, the former trend is the prototypical behavior found here away from SVP. At SVP, however, the energy balance in favor of CC grows with $L$ because the magnitude of the integrated potential energy term increases with $L$.

The general problem of behavior below SVP is more complicated because of the presence of the $\Delta$ term and the attendant possibility that a film configuration $F$ has a lower free energy than that of the capillary condensed phase. The free energy functional in the case of such films, symmetrically adsorbed on both surfaces (each of thickness $d$ ), reduces straightforwardly to

$$
\begin{gathered}
\Omega_{F}^{*}=4-D^{*}\left\{g(x)+g\left(L^{*}-1\right)-g\left(L^{*}-x\right)\right\}+2 \Delta(x-1), \\
x=d / z_{m} .
\end{gathered}
$$

It is then necessary to evaluate the minimum of this expression as a function of the variable $x$ and compare the result with the quantity $\Omega_{\mathrm{CC} *}$. Note that the difference between this free energy and that of the CC case is

$$
\Omega_{F}^{*}-\Omega_{\mathrm{CC}}^{*}=2+D^{*}\left\{g\left(L^{*}-x\right)-g(x)\right\}+2 \Delta\left(x-L^{*} / 2\right) .
$$

Here, the first term represents the extra surface energy of the film and the second represents the interaction between the solid surfaces and the atoms, which fill the gap when the $F$ to $\mathrm{CC}$ transition occurs; the third represents the free energy cost because the system is (in general) below SVP. The problem is exemplified in Fig. 6, which shows these functions for the case of ${ }^{3} \mathrm{He}$ interacting with Li. Only for small $L^{*}$ is the CC phase stable.

The general behavior seen for $\Delta \neq 0$ is expected. Increasing $D^{*}$ favors $F$ or CC phases, as opposed to the $E$ (empty) phase. Which of the condensed phases is stable depends on $L^{*}$. For large $L^{*}, F$ is typically favored because the cost of
CC (the $\Delta$ term) becomes large relative to the benefits (from the potential and the decrease in surface tension); the $F$ to $E$ transition curve is rather insensitive to $L^{*}$ because that transition occurs for such large $L^{*}$ that it nearly coincides with the transition for a single surface. Figure 2 shows how the $\mathrm{CC}$ regime shrinks, at the expense of the $F$ phase, with increasing distance from SVP (i.e., $\Delta$ ). Figures 3-5 reveal the evolution of the phases as the $D^{*}$ value changes in the case of ${ }^{3} \mathrm{He}$ (but the trends are general). For the weakest substrate shown (Cs), the $\mathrm{CC}$ regime dominates the behavior except for very large $L^{*}(>15$, i.e., $L>50 \AA)$. For large $D^{*}(\mathrm{Li}$ and $\mathrm{Au}$ ), the $F$ and $\mathrm{CC}$ regimes extend to much larger undersaturation and $F$ appears at much smaller $L^{*}(\sim 5)$ than in the Cs case.

While the shapes of the phase boundaries in Figs. 3-5 are qualitatively similar to one another, they differ in detail. There is, however, a common limiting behavior of the $F$ to $\mathrm{CC}$ transition for the case of very large $L^{*}$. In that case, where $\Delta$ is very small, one may prove that the transition line satisfies

$$
L^{*} \sim(2 / \Delta)\left[1+\left(3 D^{*} \Delta^{2} / 32\right)^{1 / 3}+\cdots\right] .
$$

\section{Thresholds for capillary condensation and film formation}

Equation (7) exhibits an absolute minimum and a corresponding threshold condition for $\mathrm{CC}$ :

$$
D_{\mathrm{CC}}^{*}=2 / g(\infty)=32 / 11 \sim 2.9 \text {. }
$$

This equation may be compared with the criterion derived previously for the wetting transition. Since [as discussed below Eq. (5)] the simple wetting model of Cheng et al. [10] is so similar to the present model, it is not surprising that a simple relation exists between the $\mathrm{CC}$ threshold and the wetting transition condition:

$$
D_{W}^{*}=2 D_{\mathrm{CC}}^{*} \sim 5.8 .
$$

This threshold substrate attraction for wetting is thus twice as large as that for $\mathrm{CC}$. The reason is simply the fact that the attractive potential contributes at both interfaces in the case of CC. A particularly intriguing test of this calculation is that of ${ }^{4} \mathrm{He} / \mathrm{Cs}$. While the single surface exhibits a wetting transition near $T=2 \mathrm{~K}$ it is predicted here that $\mathrm{CC}$ ought to occur (at $\Delta=0$ ) for all temperatures (since $D^{*}=5.5$ at $T=0$ ).

Other systems shown in Table I involve values of $D^{*}$, which lie below the 2.9 threshold at the triple point, but should exhibit CC at higher $T$. Let us suppose that we know the value of $D^{*}$ at the triple point $D_{\mathrm{tp}}^{*}$ as in Table I. From the definition of $D^{*}$, we may determine the value at any other $T$ from

$$
D^{*}(T)=D_{\text {tp }}^{*} f\left(T / T_{\text {tp }}\right),
$$

where we define a function

$$
f\left(T / T_{\text {tp }}\right)=\{\sigma / n\}_{\text {tp }} /\{\sigma / n\}_{T} .
$$

The right-hand side of this equation is a universal function of $T / T_{\text {tp }}$ if the law of corresponding states is obeyed. Then Eq. (11) may be inverted to yield an implicit relation for the CC threshold: 


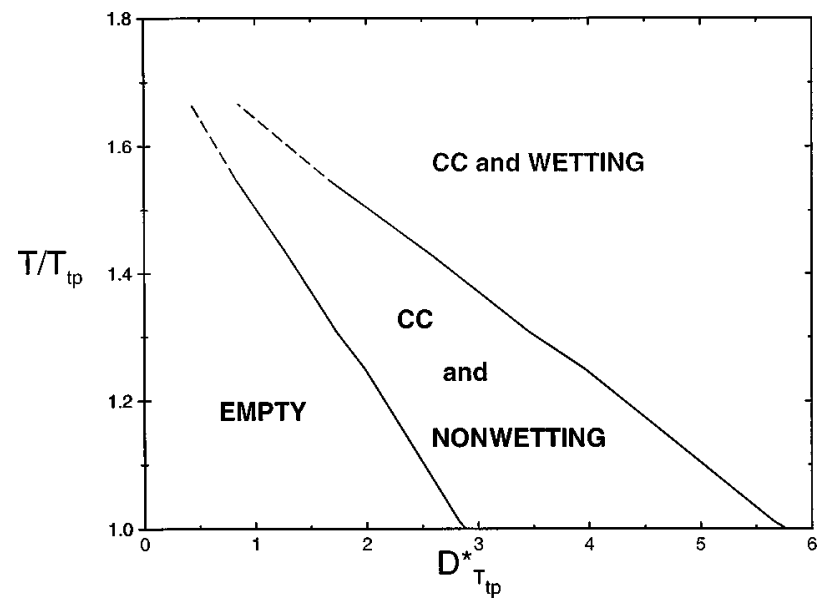

FIG. 7. Domain of temperature $T$ (relative to $T_{t}$ ) in which capillary condensation (at SVP) and/or wetting of a single surface occur, as a function of the value of $D_{\text {tp }}^{*}$ of the reduced substrate attraction at the triple point. Curves calculated from Eqs. (11)-(14), with dashes indicating limited reliability of the model near the critical temperature $\left(T / T_{\mathrm{tp}}=1.8\right)$. The curve becomes dashed when the vapor/liquid density ratio exceeds 0.1 .

$$
f\left(T / T_{\text {tp }}\right)=2.9 / D_{\text {tp }}^{*}
$$

Figure 7 displays this dependence, derived from experimental data for the density and surface tension of $\operatorname{Ar}[22,23]$. Note that even very small values of $D_{\text {tp }}^{*}$ yield CC transitions at sufficiently high $T$. One should bear in mind that temperatures approaching the critical point $\left(T / T_{\mathrm{tp}} \sim 1.8\right)$ will not be well described by our model, which neglects the vapor density in Eq. (5), assumes that the interface is discontinuous, and neglects fluctuations in general. In the regime of extremely weak substrate, for example, one expects a capillary drying transition to occur at $\Delta<0$, i.e., above SVP [13].

It is interesting that the same threshold condition applies to film formation in the slab geometry and to the wetting of a single surface [i.e., Eq. (11)]. This may be proved as follows. Since $\Delta>0$, Eq. (8) implies that if a film is stable,

$$
4-D^{*}\left\{g(x)+g\left(L^{*}-1\right)-g\left(L^{*}-x\right)\right\}<0 .
$$

Hence,

$$
D^{*}>4 /\left\{g(x)+g\left(L^{*}-1\right)-g\left(L^{*}-x\right)\right\} .
$$

We may derive an inequality for the denominator:

$$
g\left(L^{*}-1\right)-\left[g\left(L^{*}-x\right)-g(x)\right]<g\left(L^{*}-1\right)<g(\infty),
$$

where we have used $x<L / 2$ and the monotonicity of $g(x)$. Hence, we arrive at a criterion for film formation in the slab geometry:

$$
D^{*}>4 / g(\infty) \text {. }
$$

This is precisely the single surface wetting condition, Eq. (11). What is surprising prima facie about this result is that one might have expected the film formation criterion to be less stringent in the slab geometry than for a single surface. The reason why this is not true is that the single surface wetting condition permits films of infinite thickness to form.

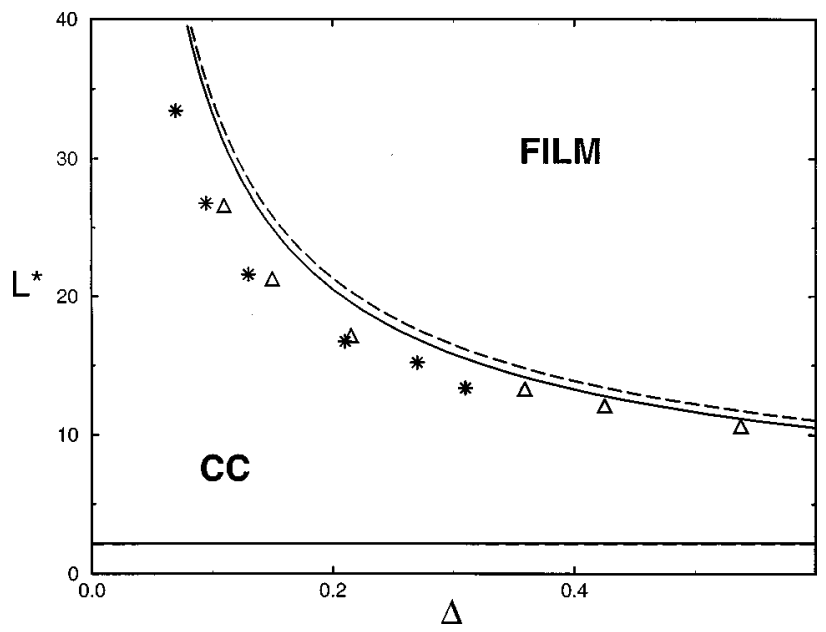

FIG. 8. Comparison between predictions of the transition from film to $\mathrm{CC}$ using the simple model of this paper (curves) and those from DF calculations of Cheng, Swift, and Cole [8] for ${ }^{4} \mathrm{He}$ : full curve and asterisks are $D^{*}=11.89$ (corresponding to $C$ $\left.=1000 \mathrm{~K}^{3}\right)$ and dashed curve and triangles are $D^{*}=14.96(C$ $=2000 \mathrm{~K}^{3}$ ).

The slab geometry sacrifices some of this benefit of the longrange potential; this loss exceeds the "gain" created by the presence of the second surface. Hence, there is no greater tendency to condense a film in the slab geometry than at a single surface.

\section{COMMENTS}

We have explored a simple model to determine the behavior expected for adsorption between parallel faces of a material. The model predicts a rich variety of phases. The phase diagrams can be understood in terms of two systemdependent parameters: a dimensionless well depth and a dimensionless gap size. This finding is remarkably simple and, therefore, esthetically appealing.

We are led to address the crucial question of whether the model is as reliable as its predecessor, which worked well in describing wetting behavior on a single surface [10]. To do so, we have embarked on a set of numerical calculations, which will be completed and described in the future. These are density-functional (DF) calculations for both isotopes of He and classical Monte Carlo simulations of Ne respectively $[17,18]$. Since we have already obtained a few results for the case of ${ }^{3} \mathrm{He}$, we may compare them with those of our simple model. Other comparisons will be presented elsewhere. Overall, the model seems to work well, as judged from the data points appearing in Figs. 3-5. In Fig. 3, there are two kinds of comparison in the case of $D^{*}=9.42$. For the case of $L^{*}=20$, one observes that the film appears in the DF calculation at nearly the same value of $\Delta$ as that predicted here. Moreover, this common value is very close to that found experimentally [12] for ${ }^{3} \mathrm{He}$ in the case of a single $\mathrm{Cs}$ surface; such a comparison is appropriate because such large $L^{*}$ films do not differ significantly from those on single surfaces. Note the possibly more interesting fact (for $L^{*}<5$ ) that the CC transition occurs in the DF results at virtually the same value of $\Delta$ as found in the simple model. This agreement is noteworthy. 
In the case of $D^{*}=20.9$, presented in Fig. 4, one finds similarly good agreement. At $L^{*}=10$, the film forms at nearly the predicted value of $\Delta$. At $L^{*}=6$, the $E$ to $F$ and $F$ to $\mathrm{CC}$ transitions also agree rather well with our simple model's predictions. Finally, for $D^{*}=88.8$, the agreement seen in Fig. 5 is also quite good for the value of $\Delta$ at the $E$ to $F$ transition.

Figure 8 compares density-functional results of Cheng Swift, and Cole [8] for $\mathrm{CC}$ of ${ }^{4} \mathrm{He}$, in the case of two different potentials. Here, too, the agreement is good.

We may summarize the results of this work as follows. An extremely simple model of capillary condensation has been explored. The resulting behavior corresponds to a universal dependence of the phase diagram (expressed in re- duced variables) on a single parameter $D^{*}$. This "corresponding states" behavior has not been recognized previously, to our knowledge. Finally, the method has been validated thus far in tests for ${ }^{3} \mathrm{He}$ and ${ }^{4} \mathrm{He}$, obtained with DF methods. More extensive tests for both these and classical fluids are in progress.

\section{ACKNOWLEDGMENTS}

We are grateful to Mary J. Bojan, Bill Steele, George Stan, Flavio Toigo, and Moses Chan for helpful discussions. This research was supported by the National Science Foundation and by the University of Buenos Aires.
[1] B. V. Derjaguin, Zh. Fiz. Khim. 14, 137 (1940) [Acta Physicochim. URSS 12, 181 (1940)].

[2] S. Dietrich, in Phase Transitions and Critical Phenomena, edited by C. Domb and J. L. Lebowitz (Academic, London, 1988), Vol. 12, p. 1.

[3] W. F. Saam and M. W. Cole, Phys. Rev. B 11, 1086 (1975); M. J. Bojan, E. Cheng, M. W. Cole, and W. A. Steele, Adsorption 2, 51 (1996); M. J. Bojan and W. A. Steele, Carbon 36, 1417 (1998).

[4] R. Evans, U. Marini Bettolo Marconi, and P. Tarazona, J. Chem. Phys. 84, 2376 (1986); R. Evans, in Liquids at Interfaces, edited by J. Charvolin, J. F. Joanny, and J. Zinn-Justin (Elsevier, Amsterdam, 1990).

[5] R. F. Cracknell, D. Nicholson, and N. Quirke, J. Chem. Soc., Faraday Trans. 90, 1487 (1994); K. E. Gubbins, M. SliwinskaBartokwiak, and S.-H. Suh, Mol. Simul. 17, 333 (1996).

[6] B. K. Peterson, K. E. Gubbins, G. S. Helfinger, U. M. B. Marconi, and F. Van Swol, J. Chem. Phys. 88, 6487 (1988); R. Radhakrishnan and K. E. Gubbins, Phys. Rev. Lett. 79, 2847 (1997).

[7] K. M. Godshalk and R. B. Hallock, Phys. Rev. B 36, 8294 (1987).

[8] E. Cheng, M. R. Swift, and M. W. Cole, J. Chem. Phys. 99, 4064 (1993).

[9] M. J. Bojan, R. V. Slooten, and W. A. Steele, Sep. Sci. Technol. 27, 1837 (1992); Q. Wang and J. K. Johnson, Int. J. Thermophys. 19, 835 (1998); Mol. Phys. 95, 299 (1998).

[10] E. Cheng, M. W. Cole, W. F. Saam, and J. Treiner, Phys. Rev. B 46, 13967 (1992); 47, 14 661(E) (1993); 48, 18214 (1993).
[11] J. Rutledge and P. Taborek, Phys. Rev. Lett. 69, 937 (1992); R. B. Hallock, J. Low Temp. Phys. 101, 31 (1995); G. Mistura, H. C. Lee, and M. H. W. Chan, ibid. 96, 221 (1994); J. E. Rutledge and P. Taborek, ibid. 95, 405 (1994).

[12] D. Ross, J. A. Phillips, and P. Taborek, J. Low Temp. Phys. 106, 81 (1997).

[13] G. B. Hess, M. J. Sabatini, and M. H. W. Chan, Phys. Rev. Lett. 78, 1739 (1997).

[14] A. Chizmeshya, M. W. Cole, and E. Zaremba, J. Low Temp. Phys. 110, 677 (1998).

[15] M. M. Calbi and E. S. Hernandez, Phys. Rev. B 57, 13258 (1998).

[16] J. Dupont-Roc, M. Himbert, N. Pavloff, and J. Treiner, J. Low Temp. Phys. 81, 31 (1990).

[17] M. M. Calbi, S. Gatica, M. W. Cole, and F. Toigo (unpublished).

[18] R. Evans, J. Phys.: Condens. Matter 2, 8989 (1990); P. B. Balbuena and K. E. Gubbins, Langmuir 9, 1801 (1993).

[19] G. Vidali, G. Ihm, H. Y. Kim, and M. W. Cole, Surf. Sci. Rep. 12, 133 (1991); L. W. Bruch, M. W. Cole, and E. Zaremba, Physical Adsorption: Forces and Phenomna (Oxford University Press, Oxford, 1997).

[20] Nonadditive corrections should be small, as discussed in M. Schmeits and A. A. Lucas, Prog. Surf. Sci. 14, 1 (1983).

[21] J. S. Rowlinson and B. Widom, Molecular Theory of Capillarity (Clarendon Press, Oxford, 1989).

[22] S.-T. Wu and G.-S. Yan, J. Chem. Phys. 77, 5799 (1982).

[23] N. B. Vargaftik, Tables on the Thermophysical Properties of Liquids and Gases (Hemisphere, Washington, D.C., 1975). 